\title{
Microbial profile of canine persistent wound infections
}

\author{
A. Padhy ${ }^{1}$, R. Mishra ${ }^{1}$, S. S. Behera ${ }^{2}$, A. R. Sahu ${ }^{3}$ and S. Sahoo ${ }^{4}$
}

1. Department of Veterinary Microbiology, College of Veterinary Science and Animal Husbandry, Orissa University of Agriculture and Technology, Bhubaneswar, Odisha, India; 2. Department of Veterinary Surgery and Radiology, College of Veterinary Science and Animal Husbandry, Orissa University of Agriculture and Technology, Bhubaneswar, Odisha, India;

3. Division of Veterinary Biotechnology, Indian Veterinary Research Institute, Bareilly, UP, India; 4. Department of Veterinary Gynaecology and Obstetrics, College of Veterinary Science and Animal Husbandry, Orissa University of Agriculture and Technology, Bhubaneswar, Odisha, India.

Corresponding author: A. Padhy, email: dearlita123@gmail.com

Received: 14-02-2014, Revised: 18-03-2014, Accepted: 21-03-2014, Published online: 16-04-2014

doi: 10.14202 /vetworld.2014.244-247

How to cite this article: Padhy A, Mishra R, Behera SS, Sahu AR and Sahoo S (2014) Microbial profile of canine persistent wound infections, Veterinary World 7(4): 244-247.

\begin{abstract}
Aim: To analyse the microbial profile of canine persistent wound infections.

Materials and Methods: The total wound samples $(n=172)$ taken from both traumatic (140) and post-surgical (32) persistent wounds in canines were processed for routine microbial isolation and identification during a period of 15 months.

Results: Staphylococcus intermedius was found to be the predominant isolate from all types of wounds under study. It was followed by Staphylococcus aureus, Pseudomonas aeruginosa, E. coli, Pasteurella spp., Corynaebacterium spp. and Bacillus spp. From different traumatic wounds of dogs, S. intermedius $(92 / 140=65.7 \%)$ and from surgical wounds, P. aeruginosa $(24 / 32=75 \%)$ were found to be the predominant isolates recovered whereas the most commonly isolated bacterial genus in both traumatic and surgical wounds of dogs was Staphylococcus spp.
\end{abstract}

Conclusion: Canine wounds are polymicrobial in nature. Hence proper microbial laboratory diagnosis and presence of multiple organisms in a wound are to be taken into consideration for effective treatment of persistent wound infections in dogs.

Keywords: canine persistent wound, microbial profile, polymicrobial.

\section{I ntroduction}

Wounds can be classified as acute and chronic. The acute wounds either traumatic or surgical move through the healing process at a predictable rate from insult to closure but chronic wounds are not resolved over a reasonable period of time no matter the cause $[1,2]$. In wounds, the presence of exposed subcutaneous tissue provides a favourable substratum for a wide variety of micro-organisms to contaminate and colonise. If the involved tissue is devitalised and the host immune response is compromised, the conditions become optimal for microbial growth [3]. It is reported that more than $80 \%$ of persistent bacterial infections are likely to involve biofilms $[4,5,6]$. The bacteria adhere to the surface of wound by attaching to the exposed extracellular matrix, multiply and develop in to microcolonies. These colonies aggregate in to larger groups known as Biofilms. These biofilms once established, resist the action of antimicrobials and evade the host defense mechanism [7]. The chronic wound infections in dogs cause economic losses to pet owners due to the cost of treatment for longer periods. For greater understanding of wound ecology its microbial analysis is necessary which may allow for better management to facilitate wound healing.

In the current study it was planned to evaluate the

Copyright: The authors. This article is an open access article licensed under the terms of the Creative Commons Attribution License (http://creativecommons.org/licenses/by/2.0) which permits unrestricted use, distribution and reproduction in any medium, provided the work is properly cited. microbial profile of persistent wounds in dogs for their better managemental practices.

\section{Materials and Methods}

Ethical approval: The approval for collecting wound samples from dogs was taken from Instituitional Animal Ethics Committee.

Collection of sample: In order to analyse the microbial profile of canine persistent wound infections, dogs of different age groups and breeds with various history of wounds of more than 16 days old infection, were included in the study. The study was carried out in the laboratory of Department of Veterinary Microbiology and Teaching Veterinary Clinical Complex (TVCC) in the college of Veterinary Science and Animal Husbandry, Orissa University of Agriculture and Technology, Bhubaneswar in the period of March 2012- June 2013. Two swabs for each sample were collected in sterile way for duplicate cultures. A total of 140 samples were collected from dogs having history like prolonged nonhealing wounds originated from accidents, infected wounds like otitis, anal wound, mammary tumor associated wounds, gangrenous tail wounds and pyoderma. Also 32 samples originated from post-surgical cases of ear haematoma, mandibular fracture and inguinal wounds were included in the study.

I solation and identification of microbes: Samples were processed for the aerobic bacterial culture by inoculating swabs in to TSB (Tryptone Soya Broth), 


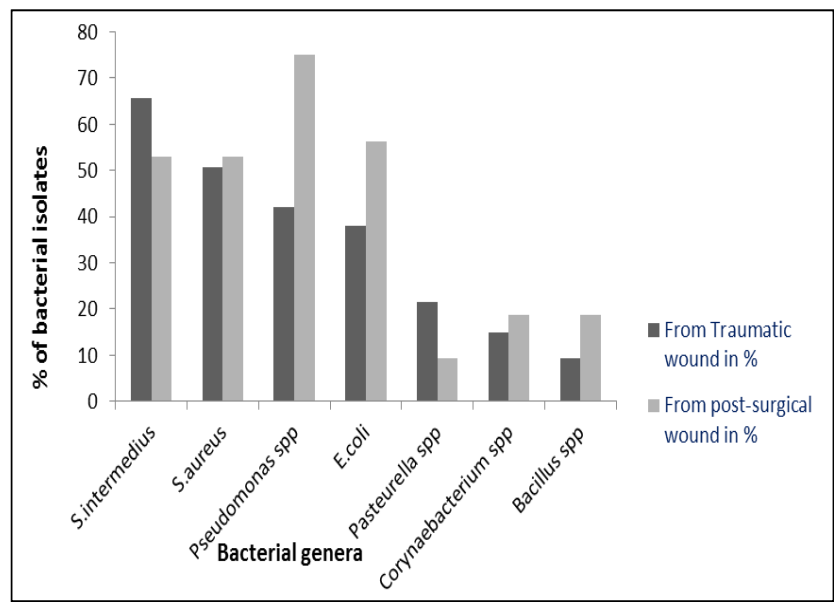

Figure-1: Graph showing percentage of bacterial genera isolated from traumatic and post-surgical wounds.

Table-1: The prevalence of different bacteria species from canine infected wounds $(n=172)$.

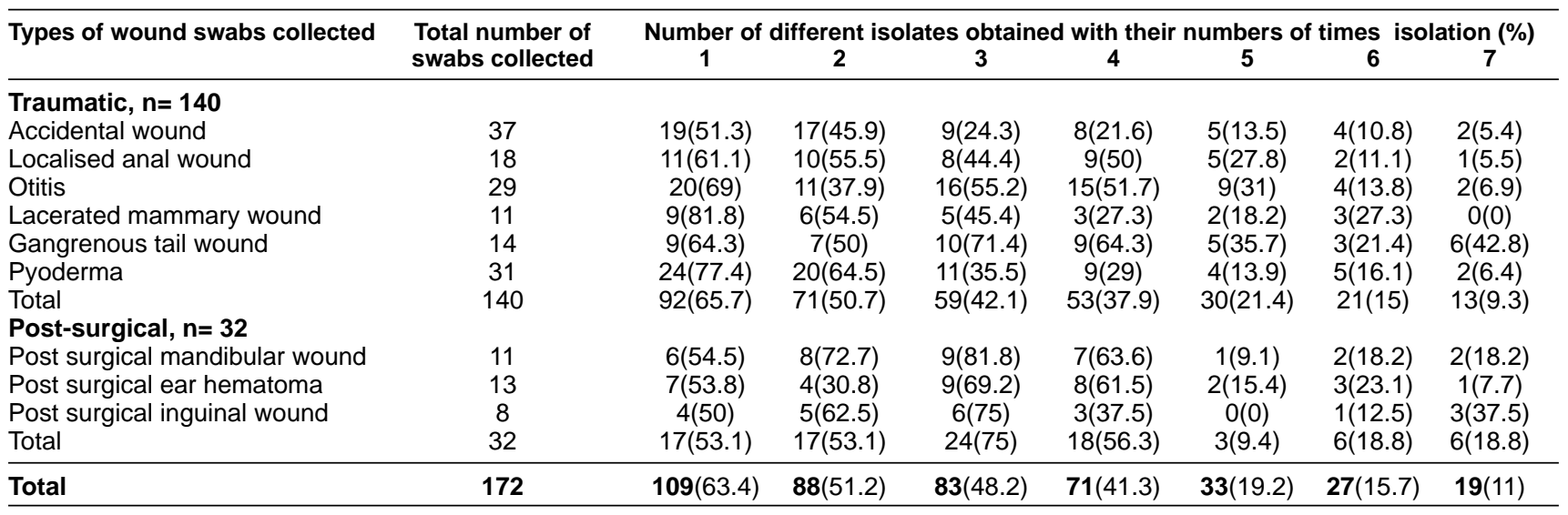

1. S. intermedius, 2. S. aureus, 3. P. aeruginosa, 4. E.coli, 5. Pasteurella spp., 6. Corynebacterium spp., 7. Bacillus spp.

and incubated at $37^{\circ} \mathrm{C}$ for $24 \mathrm{hrs}$ for growth of bacteria. By Gram staining the presence of bacteria were confirmed. To isolate colonies for each types of bacteria present in culture, they were streaked and incubated on TSA (Tryptone Soya Agar), as it provides enough nutrient for wide variety of bacteria . Then colony morphology was noted and Gram staining of each type of colony was done to confirm the morphological differences among them. Bacteria from each type of colony were taken and cultured in separate tubes having BHI broth for obtaining pure cultures. Then those were taken for identification using conventional biochemical tests like IMVC, catalase, oxidase, nitrate etc. and were cultured in suitable selective media agar plates such as Brain Heart Infusion (BHI) Broth, MLB (McConkey Lactose Broth), MLA (McConkey Lactose Agar), EMB (Eosin Methylene Blue) Agar, Mannitol Salt Agar, Pseudomonas Isolation Agar, Potassium Tellurite Agar, Nutrient Broth, Blood Agar and Nutrient Agar, obtained from HiMedia, Mumbai. [8].

\section{Results}

In the wound samples $(n=172)$ collected from various types of wound infections of dogs, the predominant isolate was found to be Staphylococcus intermedius (63.4\%). It was followed by Staphylococcus aureus (51.2\%), Pseudomonas aeruginosa (48.2\%), E. coli $(41.3 \%)$, Pasteurella spp. (19.2\%), Corynaebacterium spp. (15.7\%) and Bacillus spp. (11.0\%). The isolates obtained are depicted in Figure-1. From different types of wounds like accidental wound $(n=37)$, localized anal wound $(n=18)$, otitis $(n=29)$, lacerated mammary wound $(n=11)$, gangrenous tail wound $(n=14)$, pyoderma $(n=31)$, post- surgical mandibular wound $(n=11)$, post-surgical ear haematoma wounds $(n=13)$ and post-surgical inguinal wounds $(n=8), S$. intermedius was isolated and identified in 19 (51.3\%), 11(61.1\%), 20(81.8\%), 9(81.8\%), 9(64.3\%), 24 (77.4\%), $6(54.5 \%), 7(53.8 \%)$ and $4(50 \%)$ times respectively. Similarly the frequency of isolation of $S$. aureus, $P$. aeruginosa, E.coli, Pasteurella spp., Corynaebacterium spp. and Bacillus spp. in all types of wounds is shown in Table-1. While considering association of particular bacteria in a particular type of wound the following findings were observed. S. intermedius was a major source of contamination in all the traumatic wounds except gangrenous tail wounds where the $P$. aeruginosa was predominant. Similarly Bacillus spp. was found to be least in all the traumatic wounds except gangrenous tail wounds where Corynaebacterium spp. was least predominant. In all the post-surgical wounds $P$. aeruginosa was the major isolated pathogen whereas Pasteurella spp. in both post-surgical mandible wounds and post-surgical inguinal wounds. Bacillus 
$s p p$. in post-surgical ear hematoma were found to be least predominant. A great variation was obtained for the bacteria isolated from different types of traumatic wounds than that of post-surgical wounds. From different traumatic wounds, S. intermedius (92/140 $=65.7 \%$ ) was found as predominant isolate. In postsurgical wounds, $P$. aeruginosa $(24 / 32=75 \%)$ was the most predominant isolate recovered whereas the most commonly isolated genus in both traumatic and surgical wounds of dogs was mostly of Staphylococcus. In all types of wounds both in traumatic and post-surgical wounds mixed infections were present. Among traumatic wounds maximum mixed infection was observed in cases of gangrenous tail wounds whereas least mixed infection was in accidental wounds. Similarly in postsurgical wounds, maximum and minimum mixed infections were observed in post-surgical mandible wound and post-surgical ear haematoma respectively.

\section{Discussion}

The present study revealed that the isolates of $S$. intermedius, S. aureus, P. aeruginosa, E.coli, Pasteurella spp., Corynaebacterium spp. and Bacillus spp. were persistently causing wound infections in dogs. This suggests that canine wounds are polymicrobial in nature and containing pathogens which are significantly prevalent in chronic wounds. This agrees with the findings of previous studies $[3,9,10,11]$. It was reported by Freeman et al [12] that wounds are polymicrobial in nature in equines too. The study also showed that Staphylococcus found in synergistic relation with other bacteria in infected wounds were mostly with $E$. coli and $P$. aeruginosa. This shows similarity with the finding of Thomas [13] in which it was reported that bacteria like Staphylococcus never occurs singly and mostly found synergistically with other bacteria in infected wounds. The synergism of bacteria might lead to greater pathogenesis than individual inputs. In the present study, S. intermedius (65.7\%) was found to be the most predominant isolate from traumatic wounds which is in accordance of the earlier works $[14,15]$, who reported that most prevalent bacteria was $S$. intermedius in dog infected wounds and that it was the most common pathogen isolated in dogs having wounds arising due to dog bites .The increased prevalence of $S$. intermedius might be due to its occurrence as commensal on skin and mucus membranes in dogs.

However P. aeruginosa $(75 \%)$ was found to be the predominant isolate in postsurgical wounds which shows the similarity with previous reports in human beings [16, 17]. The genus Staphylococcus was most commonly isolated in both traumatic and post-surgical wounds. This is similar to previous reports in humans and equines $[16,18]$. An explanation for the above results might be that the microbial isolates have a strong affinity for that particular site. The composition and variety of microbial agents in wounds were influenced by numerous factors such as localisation, depth of affected tissues, tissue perfusion, quality, intensity and strength of host antibacterial immune response [14]. It has been reported that Staphylococcus serves as a major source of contamination during surgery [19] and $S$. aureus is encountered in $50 \%$ of orthopedic cases [20].

But in the present study $P$. aeruginosa was the major isolates in both post-surgical and orthopedic (mandibular wound) cases. This variation could be due to our limited study focusing only on three types of postsurgical wounds. Among different traumatic wounds mixed infection was least in accidental wound and more in gangrenous tail wound, which is chronic in nature and always presented in delayed condition after failure of primary treatment. Similarly, in post-surgical wounds, mixed infection was more in mandibular wound because oral orthopedic wound always take more time for healing and there is chance of more contamination and inability to maintain aseptic condition during healing period. In post-surgical ear hematoma cases less mixed infection was observed which might be due to presence of less soft tissue on ear pinna and more vascularity. As most wound infections involve mixed populations of both aerobic and anaerobic micro-organisms [3, 14], the complete microbial profile of canine persistent wound infections can be obtained after analyzing for anaerobes along with aerobes. However analysis for anaerobes could not be conducted in this study due to lack of facilities.

\section{Conclusion}

Total collected wound samples $(n=172)$ from persistent wound infections in dogs were processed for isolation and identification which revealed that wound microbiology of dog is complex. The microbe $S$. intermedius was found to be the most predominant isolate in traumatic wounds and $P$. aeruginosa in postsurgical wounds. The Staphylococcus genus was found to be the most common isolate from both traumatic and post-surgical wounds. The other isolates obtained in decreasing order of prevalence were E. coli, Pasteurella spp., Corynaebacterium spp. and Bacillus spp. This suggests that the canine wounds are polymicrobial in nature. The results reported in this study may serve as a guide for further studies in the same species and may help the veterinarians for proper microbial laboratory diagnosis and also for effective management of persistent wound infection in dogs.

\section{Authors' contributions}

RM planned and designed the study. SSB and SS collected samples for study, AP carried out the work and wrote the manuscript. ARS, SSB and SS helped during manuscript writing and cross checking. All authors read and approved the final manuscript.

\section{Acknow legdements}

All authors are thankful to the Director of TVCC and Dean of College of Veterinary Science and Animal Husbandry, Bhubaneswar, Odisha for providing facilities to carry out the study. The fund for the study was provided by Department of Veterinary Microbiology 
of College of Veterinary Science and Animal Husbandry, Bhubaneswar.

\section{Competing interests}

The authors declare that they have no competing interests.

\section{References}

1. Kane, D. P. (2007) Chronic wound healing and chronic wound management. In: Chronic Wound Care: A Clinical Source Book for Healthcare Professionals. 4th ed. Malvern, PA: HMP Communications, 11-23.

2. Broderick, N. (2009) Understanding Chronic Wound Healing. J. Nurse Pract, 34(10): 17-22.

3. Bowler, P.G., Duerden, B.I. and Armstrong, D.G. (2001) Wound microbiology and associated approaches to wound management. Clin Microbiol. Rev. 14: 244-269.

4. Widgerow, A.D. (2008) Persistence of the chronic woundimplicating biofilm. Wound Heal South Afr. 1(2): 5-7.

5. Davis, S.C., Ricott, C., Cazzaniga, A. and Welsh, E. (2008) Microscopic and physiologic evidence for biofilm associated wound colonization in vivo. Wound Rep Reg. 16: 23-29.

6. Costerton, J.W., Lewandowski, Z., Caldwell, D.E., Korber, D.R. and Lappin-Scott, H.M. (1995) Microbial Biofilms. Annu Rev Microbiol.49: 711-45.

7. Wolcott, R.D. and Rhoads, D.D. (2008) A study of biofilmbased wound management in subjects with critical limb ischaemia. J Wound Care. 17: 145-155.

8. Quinn, P. J., Carter, M. E., Markey, B. K., Carter, G. R. (1999) Section : 2 Bacteriology. In : Clinical Veterinary Microbiology, Harcourt Publisher Limited, p118-254.

9. Bryan, C.S., Parisi, J.T. and Strike, D.G. (1987) Vertebral osteomyelitis due to Staphylococcus warneri attributed to a hick man catheter. Diagn. Microbiol. Infect. Dis. 8: 57- 59.
10. Wood, C.A., Sewell, D.L. and Strausbaugh, L.J. (1989) Vertebral osteomyelitis and native valve endocarditis caused by Staphylococcus warneri. Diagn. Microbiol. Infect. Dis. 12 (3): 261-263.

11. Abrahamian, M.F. and Golstein, J.C.E. (2011) Microbiology of Animal Bite Wound Infections. Clin. Microbiol. Rev. 24(2): 231-246.

12. Freeman, K., Woods, E., Welsby, S., Percival, S.L. and Cochrane, C.A. (2009) Biofilm evidence and the microbial diversity of horse wounds. Canadian Journal of Microbiology. 55:197-202.

13. Thomas J.G. (2008) Advancing your practice: Understanding Wound infection and the Role of Biofilms. Association for the Advancemnent of Wound Care. 2-9.

14. Urumova, V., Chaprazov, T.S., Lyutskanov, M. and Borisov, I. (2012) Microbiological analyses of canine infected wounds. Revue Méd.Vet.163(4): 201-205.

15. Kelly, P.J., Mason, P.R., Els, J. and Matthewman, L.A. (1992) Pathogens in dog bite wounds in dogs in Harare, Zimbabwe. Vet. Rec. 131:464-466.

16. Masaadeh, H.A. and Jaran, A.S. (2009) Incident of Pseudomnas aeruginosa in Post-Operative Wound Infection. Ame J Infec Dis. 5(1): 1-6.

17. Ruhil, K., Arora, B. and Adlakha, H. (2009) Pseudomonas aeruginosa Isolation of Post-operative Wound in a Referral Hospital in Haryana. India. J Infect Dis Antimicrob Agents. 26(2): 43-48.

18. Westgate, S.J., Percival, S.L., Knottenbelt, D.C., Clegg, P.D. and Cochrane, C.A. (2011) Microbiology of equine wounds and evidence of bacterial biofilms. Vet Microbiol. 150: 152-159.

19. Clark, C.H. (1980) Prophylactic use of antibiotic in surgery. Part 1. Mod. Vet. Pract. 1:30-33.

20. Mandal, S.P., Osbome, G.V., Mandal, A. and Percival, A. (1980) Gross infections in orthopaedic operation theatres. Indian J. Surg. 2: 60-69.

$* * * * * * * *$ 